\title{
Profil de consommation alimentaire des ménages dans la région de Mopti en Février 2016.
}

\section{Food profile of consumption household in the area of Mopti in February 2016.}

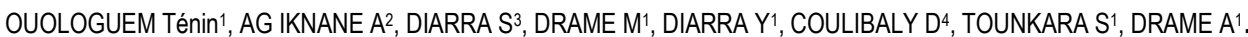

${ }^{1}$ Medecins nutritionnistes, Faculté de Faculté de médecine, Université des sciences, des techniques et technologies de Bamako, (Mali).

2 Professeur en santé Publique-nutrition, Faculté de Pharmacie, USTTB, Bamako (Mali

${ }^{3}$ Medecin épidemiologiste, Système d'Alerte Précoce,Bamako (Mali)

${ }^{4}$ Assistante en Nutrition, Faculté de Pharmacie, USTTB, Bamako (Mali).

\section{Résumé}

L'alimentation est influencée par les caractéristiques sociodémographiques et économiques. C'est dans ce contexte que la présente étude d'évaluation de la qualité du régime alimentaire des ménages et les facteurs associés dans la région de Mopti en Février 2016 a été réalisée.

II s'agissait d'une analyse secondaire des données de l'Enquête Nationale de Sécurité alimentaire et nutritionnelle (ENSAN) de Février 2016 du Mali pour la région de Mopti. La méthodologie de base était le sondage en grappe à deux degrés. L'analyse des données a été effectuée sur (SPSS) version 21.0. Des tests de khi-carré de Pearson et de régression logistique ont été réalisés avec un niveau de confiance de $95 \%$.

Notre étude avait concerné 148176 ménages à $91 \%$ ruraux et $99,2 \%$ de résidents. Les chefs de ménages (CM) étaient très majoritairement des hommes (93,5\%), mariés $(93,9 \%)$. Près de $46 \%$ des hommes CM étaient sans instruction contre $76 \%$ de femmes. Le score de consommation alimentaire (SCA) des ménages était acceptable dans $67 \%$ et un score de diversité alimentaire (SDA) de 95,9\%.Le régime quotidien était composé de céréales, légumes, huiles et sucres. Les ménages à diversité faible consommaient les céréales et huiles. Ceux à diversité moyenne consommaient en plus des légumes et du sucre. Ceux à diversité élevée consommaient en plus, des légumineuses, viandes/abats et lait. Le SCA et le SDA étaient statiquement liés $(p=0,000)$ au niveau socioéconomique, au milieu de résidence, au niveau d'instruction, au statut matrimonial et l'âge du chef de ménage. Le SCA était lié au statut du ménage et au sexe du CM.

Le régime était monotone dans l'ensemble de la région, d'où l'intérêt de renforcer la sensibilisation des ménages à une diversification de la consommation alimentaire.

Mots clés : Profil, consommation alimentaire, ménages, Mopti

\footnotetext{
Abstract

Diet is influenced by sociodemographic and economic characteristics. It is in this context that this study of assessment of the quality of household diet and associated factors in the Mopti region in February 2016 was conducted.

This was a secondary analysis of data from the February 2016 Mali National Food Security and Nutrition Survey (ENSAN) for the Mopti region. The basic methodology was the two-stage cluster survey. Data analysis was
}

performed on (SPSS) version 21.0. Pearson chi-square tests and logistic regression were performed with a 95\% confidence level.

Our study involved 148176 households at $91 \%$ rural and $99.2 \%$ residents. Heads of households (CM) were overwhelmingly men $(93.5 \%)$ married $(93.9 \%)$. Nearly $46 \%$ of $\mathrm{CM}$ men were uneducated compared to $76 \%$ of women. The dietary consumption score (SCA) of households was acceptable in $67 \%$ and a dietary diversity score (SDA) of $95.9 \%$.The daily diet consisted of cereals, vegetables, oils and sugars. Low diversity households consumed cereals and oils. Those with medium diversity also consumed vegetables and sugar. Those with high diversity also consumed legumes, meat / offal and milk. The SCA and SDA were statistically related $(p=0.000)$ at the socio-economic level, place of residence, educational level, marital status, and age of head of household. The SCA was related to the status of the household and the sex of the CM.

The diet was monotonous throughout the region, hence the importance of increasing the awareness of households to diversify food consumption.

Key words: Profile, food consumption, households, Mopti.

\section{Introduction}

La sécurité alimentaire existe lorsque toute personne, en tout temps, a un accès physique, économique et sociale à une nourriture suffisante, saine et nutritive lui permettant de satisfaire ses besoins énergétiques et ses préférences alimentaires pour mener une vie saine et active [1].

Actuellement, les questions qui touchent la consommation alimentaire se posent encore avec acuité aussi bien dans les pays dits industrialisés que dans ceux du tiers-monde [2].

La fragilité du marché international et la volatilité des prix des denrées alimentaires constituent également un risque pouvant diminuer encore plus le pouvoir d'achat des populations à faible revenu, et ainsi compromettre davantage la qualité de leur alimentation et leur statut nutritionnel [3].

L'Afrique subsaharienne est la partie du continent la plus diminue, notamment en matière économique et la plus mouvementée politiquement : en effet, elle est l'une des régions les plus pauvres au monde, et les plus sous alimentées, mais néanmoins, cela n'affecte que le trois quart des pays industrialisés. [4]

L'analyse de situation alimentaire en Afrique subsaharienne montre un écart croissant entre les 
besoins de consommation et de nutrition et les disponibilités alimentaires au niveau global des pays. La faiblesse des gains de productivité dans la production alimentaire et des capacités d'importation constituent les contraintes majeures à la réalisation de la sécurité alimentaire dans la région. Cependant l'augmentation de la productivité agricole (priorité budget terre) doit confronter des contraintes d'ordre naturel, socio économique. [5]

Puisque les nutriments essentiels dont nous avons besoin ne sont pas tous contenus dans un seul aliment, consommer une variété d'aliments est un élément clé d'un régime de bonne qualité. Au niveau international $[5,6]$, les directives alimentaires recommandent de consommer une variété suffisante d'aliments, ce qui est supposé assurer des apports adéquats en nutriments essentiels pour être en bonne santé.

Dans les pays en développement, l'augmentation de la variété des aliments ou groupes d'aliments consommés est essentielle où les carences en nutriments sont des problèmes majeurs [7]. Le manque de diversité alimentaire est un problème particulièrement grave chez les populations pauvres des pays en développement dont l'alimentation quotidienne est généralement basée sur une source d'amidon (céréales, tubercules) accompagnée d'une ou deux composantes supplémentaires [7]. Ce type de régime a tendance à être pauvre en plusieurs micronutriments et ceux qu'il contient ont souvent une faible biodisponibilité [7].

Dans le domaine de la nutrition, il y a un consensus selon lequel l'adoption d'une alimentation suffisamment diversifiée serait la solution idéale pour assurer les besoins en nutriments [8]. Dans les pays en développement, l'accès économique demeure un obstacle important à la consommation d'une alimentation saine [8].

Le Mali est un pays sahélien, enclavé, et structurellement vulnérable à l'insécurité alimentaire et à la malnutrition [9]. L'économie est fortement dépendante du secteur primaire: l'agriculture, l'élevage, la chasse et la sylviculture occupant $68.0 \%$ de la population active [9]. Ce secteur est lui-même tributaire de facteurs exogènes, principalement d'ordre climatique, telles les sécheresses récurrentes [9].

Les conditions de vie dans le pays continuent de subir l'impact négatif de la situation sécuritaire instable et des effets des aléas climatiques principalement dans les régions nord du pays. Les ménages maliens, déjà fortement affectés par une série de crises ont ainsi fait face à d'importantes pertes au niveau des moyens de subsistance (bétail, récoltes, sources de revenus, emploi, réduction des activités économiques, etc.). En raison de la baisse de la production agro-sylvo-pastorale par endroit et de l'impact de l'insécurité sur les circuits d'approvisionnement et les activités génératrices de revenus, plusieurs communes de la région ont des difficultés [10].
C'est dans ce contexte que la présente étude d'évaluation de la qualité du régime alimentaire des ménages et les facteurs associés dans la région de Mopti a été réalisée en Février 2016.

\section{Matériels et méthode:}

Une analyse secondaire des données de l'Enquête Nationale de Sécurité alimentaire et nutritionnelle (ENSAN) de Février 2016 du Mali a été réalisée pour la région de Mopti. La méthodologie de base était le sondage en grappe à deux degrés. Elle avait concerné 148176 ménages dans tous les cercles de la région dont la qualité du régime alimentaire a été évaluée à travers les scores de consommation et de diversité alimentaires et les facteurs associés. L'analyse des données a été effectuée à l'aide du logiciel SPSS version 21.0. Des tests statistiques de khi-carré de Pearson et de régression logistique ont été réalisés avec un degré de signification de $5 \%$.

\section{Résultats :}

Notre étude avait concerné $91 \%$ de ménages ruraux dont $99,2 \%$ de résidents. La taille moyenne des ménages était de 13,97 + ou - 10,79 personnes. Les chefs de ménages (CM) étaient en majorité des hommes $(93,5 \%)$, mariés $(93,9 \%)$ dont $55 \%$ de monogames et $38,9 \%$ de polygames. Près de $46 \%$ des hommes chefs de ménages étaient sans instruction contre $76 \%$ de femmes. L'âge moyen des chefs de ménages était de 52,51 + ou 13,68 ans. Les ménages appartenant au quintile des plus pauvres représentaient $14 \%$ contre $20,3 \%$ de ceux du quintile des plus riches.

Le score de consommation alimentaire (SCA) des ménages était acceptable dans $67 \%$ et un score de diversité alimentaire (SDA) de 95,9\%. Le nombre moyen de groupes d'aliments consommés la veille était de 6,85. Le régime alimentaire quotidien était essentiellement composé de céréales $(99,9 \%)$, huiles $(95,1 \%)$, sucres $(80,2 \%)$, légumes $(70,3 \%)$ et épices condiments $(97,1 \%)$ avec des disparités dans certaines localités. Les œufs $(3,5 \%)$, les fruits $(8,1 \%)$ étaient les moins consommés. Les ménages à diversité faible consommaient les céréales et huiles. Ceux à diversité moyenne consommaient en plus des légumes et du sucre. Ceux à diversité élevée consommaient en plus, des légumineuses, viandes/abats et lait.

Après ajustement, toutes les caractéristiques étaient statistiquement associées à la consommation alimentaire $(p=0,000)$. Cependant le milieu urbain $(\mathrm{ORa}=1,407)$, les non résidents $(\mathrm{ORa}=1,169)$, le sexe féminin (ORa = $2,668)$, les 46 ans et plus ( $\mathrm{ORa}=1,149)$, les $\mathrm{CM}$ moyens $(\mathrm{ORa}=1,071)$ et les riches $(\mathrm{ORa}=1,668)$ avaient plus de risque d'avoir une consommation alimentation inadéquate. Par contre, les non mariés $(\mathrm{ORa}=0,262)$ et les instruits $(\mathrm{ORa}=0,916)$ avaient plus de chance d'avoir une consommation alimentaire adéquate. Egalement, toutes les caractéristiques sauf le sexe du CM étaient statistiquement associées à la diversité alimentaire $(p=$ $0,000)$. Cependant le milieu urbain $(\mathrm{ORa}=2,851)$, les 
instruits (ORa $=4,077)$ avaient plus de risque d'avoir une alimentation non diversifiée. Par contre, les 46 ans et plus $(\mathrm{ORa}=0,519)$, les non mariés $(\mathrm{ORa}=0,298)$, les $\mathrm{CM}$ moyens $(\mathrm{ORa}=0,310)$ et les riches $(\mathrm{ORa}=0,546)$ avaient plus de chance d'avoir une alimentation diversifiée.

\section{Commentaires et discussion :}

Les ménages dirigés par des hommes étaient nettement supérieurs $(93,5 \%)$ à ceux dirigés par des femmes $(6,5 \%)$. Les proportions de femmes chefs de ménages étaient plus importantes dans les cercles de Mopti et Tenenkou avec respectivement $16,5 \%$ et $12,1 \%$. Ce résultat est comparable à ceux de KONE CT en 2012 dans le cercle de Nioro du sahel et SYLLA F en 2014 dans le cercle de Nara qui trouvent respectivement $99,0 \%$ et $96,6 \%$ de ménages dirigés par les hommes $(11,12)$ et différent à celui d'ENSAN Sénégal 2016 qui trouve que $70 \%$ de ménages dirigés par les hommes (13).

Selon notre étude, l'âge moyen des chefs de ménages était 52,51 ans + ou -13,68. Ce résultat est comparable à celui MUTEBA K D en 2014 à Kinshasa qui trouve que les chefs de ménages avaient une moyenne d'âge de 51 ans (2).

Dans notre étude, les chefs de ménages étaient généralement mariés (93,9\%), avec $55 \%$ de monogames et $38,9 \%$ de polygames. Ce résultat est différent de celui d'ENSAN Sénégal 2016 qui trouve que chefs de ménages étaient mariée dans $86 \%$ des cas dont $53 \%$ de monogames et $33 \%$ de polygames (13). Cela pourrait s'expliquer par la tradition, la coutume ou la religion de notre pays particulièrement dominante dans la région de Mopti. Les résultats de notre étude montraient que les chefs de ménages avaient un faible niveau d'instruction avec $45,8 \%$ de non instruits. Ce taux est différent de ceux de SYLLA F en 2014 dans le cercle de Nara (12) et de World Food Programme au Burundi en 2015 (14) qui trouvent respectivement $67,5 \%$ et $50 \%$ et différent de celui d'ENSAN Sénégal de 2016 qui trouve que 33\% des chefs de ménages sans aucun niveau d'instruction (13). Nous avons trouvé que les chefs de ménages hommes sans instruction étaient minoritaires $(44 \%)$ par rapport à ceux des femmes (76\%). Cela pourrait s'expliquer par le fait que depuis l'enfance, la scolarisation des enfants en général est faible et en particulier celle des filles.

Notre étude a révélé que $14 \%$ des ménages étaient plus pauvres contre $20,3 \%$ de plus riches. Cela pourrait s'expliquer par le manque d'emploi, la non diversité des activités génératrices de revenu, le retrait des touristes de cette localité en raison de l'insécurité.

Selon notre étude, le score de consommation alimentaire des ménages était acceptable dans près $67 \%$ des cas. II est comparable à celui d'ENSAN Mali 2015 qui trouve $70,5 \%$ au niveau national (15) et différent de celui d'ENSAN Sénégal 2016 qui trouve 83,1\% (13). Ce résultat pourrait s'expliquer par la disponibilité des récoltes en cette période de l'année.
Notre analyse a montré que le cercle de Youwarou avait le taux le plus élevé de score de consommation alimentaire avec $87,9 \%$. Ce taux est différent à ceux de KONE CT en 2012 dans le cercle de Nioro du sahel et de SYLLA F en 2014 dans le cercle de Nara qui trouvent respectivement $95,3 \%$ et $90,1 \%(11,12)$.

Près de la totalité des ménages de notre étude $(95,9 \%)$ avaient une alimentation diversifiée. Ce taux est comparable à celui d'ENSAN Mali 2015 qui trouve au niveau national $94,2 \%$ (15) et différent à celui de SYLLA F. 2014 dans le cercle de Nara (12) et d'ENSA Haïti de 2011 (16) qui trouvent respectivement $65,9 \%$ et $78 \%$. Cela pourrait s'expliquer par la crise alimentaire et sécuritaire qu'avait connue Haïti.

Selon notre étude, le nombre moyen de groupes d'aliments consommés la veille de l'enquête était de 6,85 . Le nombre moyen le plus élevé de groupes d'aliments consommés la veille de l'enquête était de 7,43 à Youwarou. Ce résultat est comparable à ceux d'ENSAN Mali en 2015 (15) et d'Elise P. en 2015 à Haïti (17) qui trouvent respectivement 6 et 6,3 groupes d'aliments consommés.

La base des repas quotidiens dans notre étude était essentiellement composée de céréales, légumes, matières grasses, sucres et condiments, avec des disparités dans les cercles. Ce résultat est comparable à ceux d'ENSAN Mali 2015 qui trouve la même composition (15), d'ENIAM Burkina de Décembre 2009 (18) qui trouve que le régime alimentaire se caractérise par une consommation quasi-générale de céréales, condiments et légumes feuilles. Elise P. en 2015 à Haïti (17) trouve que la majorité des ménages favorisent la consommation d'aliments de base qui composent un plat typique tels que les racines et tubercules, les produits céréaliers, les corps gras, les épices et condiments et les breuvages. AGVSAN au Bénin en 2008 quant à lui trouve que les aliments de base (céréales \& tubercules) sont consommés par quasiment tous les ménages (19).

Notre étude a montré un lien statistiquement significatif entre la consommation et la diversité alimentaires et les caractéristiques sociodémographiques et économiques $(p=0,000)$.

Par rapport à la consommation alimentaire, les CM femmes avaient un risque d'avoir une consommation alimentaire inadéquate par rapport aux hommes CM $\left(O R_{a}=2,668\right.$ et $\left.I C=2,501-2,849\right)$. Ce résultat est similaire à celui de Gaudin VL à Québec en 2012 (20) qui trouve que le sexe féminin a un plus grand risque que les hommes d'avoir une consommation alimentaire inadéquate $\left(O R_{a}=1,1\right.$ et $\left.I C=0,50-2,40\right)$.

La consommation alimentaire était inadéquate chez les $\mathrm{CM}$ de 46 ans et plus comparée à ceux de moins de 46 ans $\left(O R_{a}=1,149\right.$ et $\left.I C=1,120-1,173\right)$. Ce résultat est comparable à celui de Gaudin VL. à Québec en 2012 (20) qui trouve que les 40 ans et plus ont un grand risque par rapport aux moins de $40 \mathrm{ans}\left(\mathrm{OR}_{\mathrm{a}}=4,50\right.$ et $\mathrm{IC}=2,47-8,26)$. 
Les CM instruits avaient plus de chance d'avoir une consommation alimentaire adéquate par rapport aux $\mathrm{CM}$ non instruits $\left(\mathrm{OR}_{a}=0,916\right.$ et $\left.\mathrm{IC}=0,886-0,946\right)$. Ce qui est en accord avec celui de Gaudin VL. à Québec en 2012 (20) qui trouve que les instruits ont une consommation alimentaire adéquate par rapport aux non instruits $\left(\mathrm{OR}_{\mathrm{a}}=0,87\right.$ et $\left.\mathrm{IC}=0,45-1,69\right)$.

Quant à la Diversité alimentaire, notre étude a montré qu'on diversifiait moins l'alimentation en milieu urbain $\left(O R_{a}=2,851\right.$ et $\left.I C=2,412-3,369\right)$ qu'en milieu rural. $\mathrm{Ce}$ résultat est différent à celui de Bertin et al (22) qui trouve que l'alimentation est plus diversifiée en milieu urbain qu'en milieu rural $\left(\mathrm{OR}_{\mathrm{a}}=0,80\right.$ et $\left.\mathrm{IC}=0,53-1,21\right)$. Cela pourrait s'expliquer par la méthodologie utilisée, le milieu d'étude, la population de l'étude.

Les CM de 46 ans et plus avaient une alimentation plus diversifiée $\left(O R_{a}=0,519\right.$ et $\left.\mathrm{IC}=0,486-0,554\right)$ comparés à ceux de moins de 46 ans. Ce résultat est comparable à celui de Bertin et al (21) qui trouve que l'alimentation est plus diversifiée chez les $35-54$ ans que chez les moins de 35 ans $\left(O R_{a}=2,68\right.$ et $\left.I C=2,50-6,62\right)$

L'alimentation était plus diversifiée chez les CM non mariés comparés à ceux mariés $(O R=0,262$ et $I C=0,246$ $0,279)$. Ce résultat est en désaccord avec celui d'Elise P. en 2015 à Haïti (17) qui ne trouve aucun lien statistiquement significatif entre le statut matrimonial et la diversité alimentaire $(p=0,948)$.

Les CM instruits avaient un grand risque d'avoir une alimentation non diversifiée par rapport à ceux instruits $(\mathrm{OR}=4,077$ et $\mathrm{IC}=3,532-4705)$. Ce résultat est différent à ceux de Bertin et al (21) et d'Elise P. (17) qui trouvent respectivement que les instruits ont une alimentation plus diversifiée que les non instruits $\left(\mathrm{OR}_{a}=0,31\right.$ et $\mathrm{IC}=0,15$ $0,55)$ et $\left(O R_{a}=0,235\right.$ et $\left.I C=0,199-1,011\right)$. Cela pourrait s'expliquer par la méthodologie utilisée, le milieu d'étude, la population de l'étude.

\section{Conclusion}

La consommation et la diversité alimentaires étaient liées au bien-être des ménages mesuré à travers le niveau socioéconomique. Le régime alimentaire était monotone dans l'ensemble de la région, d'où l'intérêt de renforcer la sensibilisation des ménages à une diversification de la consommation alimentaire en mettant un accent particulier sur les cercles de Bankass, Djenné et Bandiagara qui présentaient les taux les plus élevés de faible diversité.

\section{Références}

1. Comité de la Sécurité Alimentaire Mondiale, S'entendre sur la terminologie, CSA, 39e session,Octobre2012,17p. https://www.bing.com/search/ (consulté le 12/11/2017)

2. MUTEBA KD. Caractérisation des modes de consommation alimentaire des ménages à Kinshasa : Analyse des interrelations entre modes de vie et habitudes alimentaires. (Thèse de doctorat). Université de LiègeGembloux-Agro-Bio Tech, Belgique 2014,179p. https://www.google.ml/search/ (consulté le 15/11/2017)

3. Brinkman, H, De Pee, S, Sanogo, I, Subran, L. et Bloem, M. W. (2010). High food prices and the global financial crisis have reduced access to nutritious food and worsened nutritional status and health. Journal of Nutrition, 140(1), 153S-161S.

4. Melgar-Quinonez, H. R., Zubieta, A. C., MkNelly, B., Nteziyaremye, A., Gerardo, M. F. D. et Dunford, C. (2006). Household food insecurity and food expenditure in Bolivia, Burkina Faso, and the Philippines. Journal of Nutrition, 136(5), 1431S-1437S.

5. US. Department of Agriculture Human Nutrition Information Service. Food Guide Pyramid: A guide to daily food choices. Home and Garden Bulletin No. 249. Washington, D.C.: U.S. Departments of Agriculture and Health and Human Resources, 1992, http://journals.sagepub.com/doi/ (consulté de 03/10/2017).

6. WHO. Preparation and use of food-based dietary guidelines. WHO Technical report, series 880 . Report of a joint FAO/WHO Consultation. Geneva: WHO, 1996.

7. http://www.microsofttranslator.com/ consulté le 03/10/ 2017.

8. Popkin BM. Urbanization, lifestyle changes and the nutrition transition. World Development 1999; 27(11): 1905-16. https://www.bing.com/search/ (consulté le 03/10/ 2017)

9. Torheim, LE, Ferguson, EL, Penrose $\mathrm{K}$ et Arimond M. (2010). Women in resourcepoor settings are at risk of inadequate intakes of multiple micronutrients. Journal of Nutrition, 140(11), 2051S-2058S.

10. SAP, WFP, FAO, FEWS NET. Enquête Nationale sur la Sécurité Alimentaire et Nutritionnelle (ENSAN) Mali : Rapport de synthèse, Mars 2016, (Document électronique)

11. Mali : Région de Mopti : Etat des lieux de la situation alimentaire 26 mai 2015

12. http://maliactu.net/mali-region-de-mopti-etat-des-lieux-dela-situation-alimentaire/ Consulté le 21/11/2017

13. KONE CT. La sécurité alimentaire des ménages du cercle de Nioro du Sahel, Thèse de Médecine, Université de Bamako, 2014, 90p.

14. SYLLA F Sécurité alimentaire et nutritionnelle des enfants de 6 à 69 mois et les femmes en âges de procréer dans le cercle de Nara, Thèse de Médecine, Université de Bamako, 2014, 74p.

15. Secrétariat Exécutif du Conseil de Sécurité Alimentaire du Sénégal, PAM et Word Vision: Enquête Nationale de Sécurité Alimentaire et Nutritionnelle (ENSAN). Rapport définitif, Sénégal 2016.

16. Ministère de l'Agriculture et de l'Elevage, PAM Burundi : système de suivi de la sécurité alimentaire, World Food Programme - Burundi Décembre 2015. https://www.bing.com/search/ (consulté le 13/11/18)

17. SAP, WFP FAO FEWS NET et CLUSTER SECURITE ALIMENTAIRE. Enquête Nationale sur la Sécurité Alimentaire et Nutritionnelle (ENSAN) Mali : Rapport de synthèse Mali, 2015

18. Coordination nationale de la sécurité alimentaire. Enquête nationale de la sécurité alimentaire (ENSA), Haïti, 2011. http://www.cnsa509.org/Web/Etudes/Rapport/ (consulté le 13/11/18)

19. Elise $P$. La qualité de l'alimentation et l'accès alimentaire des ménages vulnérables habitant dans une zone d'intervention d'agriculture de santé publique en Haïti, Thèse, Université d'Ottawa, Canada 2015, 180p.

20. Direction Générale de la Promotion de l'Economie Rurale : Enquête Nationale sur l'Insécurité Alimentaire et la Malnutrition (ENIAM) Burkina, rapport définitif, Décembre 2009 
21. Gouvernement du Bénin, UNICEF et PAM: Analyse Globale de la Vulnérabilité de la Sécurité Alimentaire et Nutritionnelle (AGVSAN). Bénin en 2008

22. Gaudin VL. Facteurs associés à l'alimentation traditionnelle au sein de trois communautés cries du Nord du Québec. Université de Montréal, Canada 2012,178p.
23. Bertin M., Touvier M., Dubuisson C., Dufour A., Havard S., Lafay L., Volatier J.-L., Lioret S. (2016) Dietary patterns of French adults: associations with demographic, socioeconomic and behavioural factors. Journal of Human Nutrition and Dietetics, 29 (2), 241-254.

Tableau : Caractéristiques sociodémographiques et économiques associés à la consommation et la diversité alimentaires dans la région de Mopti en Février 2016.

\begin{tabular}{|c|c|c|c|c|c|c|}
\hline \multirow[b]{2}{*}{ Variables } & \multicolumn{3}{|c|}{ Consommation alimentaire inadéquate } & \multicolumn{3}{|c|}{ Alimentation non diversifiée } \\
\hline & $\mathrm{OR}_{\mathrm{a}}$ & $\mathbf{I}_{95 \%}$ & P-value & $\mathrm{OR}_{\mathrm{a}}$ & $\mathrm{IC}_{95 \%}$ & P-value \\
\hline \multicolumn{7}{|c|}{ Milieu de résidence } \\
\hline Rural & 1 & & & 1 & & \\
\hline Urbain & 1,407 & $1,350-1,467$ & 0,000 & 2,851 & $2,412-3,369$ & 0,000 \\
\hline \multicolumn{7}{|c|}{ Statut du Ménage } \\
\hline Résidents & 1 & & & & & \\
\hline Non résidents & 1,169 & $1,029-1,329$ & 0,000 & 1 & 1 & 1 \\
\hline \multicolumn{7}{|l|}{ Sexe } \\
\hline Masculin & 1 & & & & & I \\
\hline Féminin & 2,668 & $2,501-2,849$ & 0,000 & 1 & 1 & \\
\hline \multicolumn{7}{|l|}{ Tranche d'âge } \\
\hline $20-45$ ans & 1 & & & 1 & & \\
\hline 46 ans et plus & 1,149 & $1,120-1,173$ & 0,000 & 0,519 & $0,486-0,554$ & 0,000 \\
\hline \multicolumn{7}{|c|}{ Statut Matrimonial du CM } \\
\hline Mariés & 1 & & & 1 & & \\
\hline Non Mariés & 0,262 & $0,246-0,279$ & 0,000 & 0,298 & $0,264-0,337$ & 0,000 \\
\hline \multicolumn{7}{|c|}{ Niveau d'instruction CM } \\
\hline Non Instruits & 1 & & & 1 & & \\
\hline Instruits & 0,916 & $0,886-0,946$ & 0,000 & 4,077 & $3,532-4705$ & 0,000 \\
\hline \multicolumn{7}{|c|}{ Bien être économique } \\
\hline Pauvres & 1 & & & 1 & & \\
\hline Moyens & 1,071 & $1,040-1,103$ & 0,000 & 0,310 & $0,288-0,334$ & 0,000 \\
\hline Riches & 1,668 & $1,626-1,710$ & 0,000 & 0,546 & $0,509-0,586$ & 0,000 \\
\hline
\end{tabular}

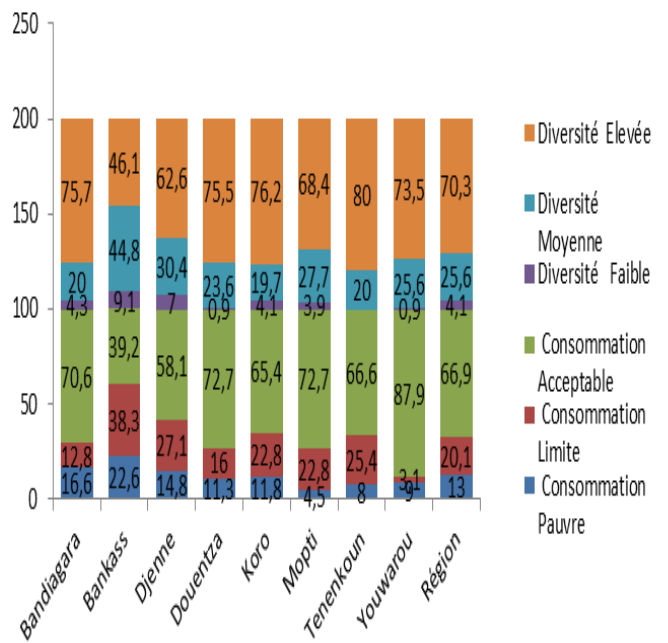

Figure 1: Répartition des cercles selon les scores de consommation et de diversité alimentaires

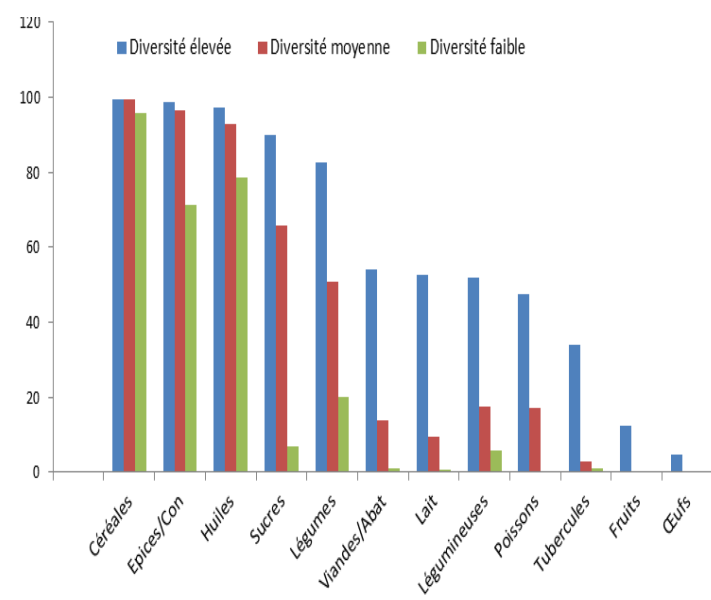

Figure 2: Fréquence de consommation des groupes alimentaires selon les catégories de diversité (en \%) 